\title{
Continuum bound states as surface states of a finite periodic system
}

\author{
D. W. L. Sprung, P. Jagiello, and J. D. Sigetich \\ Department of Physics and Astronomy, McMaster University, Hamilton, Ontario, Canada L8S 4M1
}

J. Martorell

Departament d'Estructura i Constituents de la Materia, Facultat Física, University of Barcelona, Barcelona 08028, Spain

(Received 4 October 2001; revised 19 July 2002; published 28 February 2003)

\begin{abstract}
We discuss the relation between continuum bound states (CBSs) localized on a defect, and surface states of a finite periodic system. We model an experiment of Capasso et al. [F. Capasso, C. Sirtori, J. Faist, D. L. Sivco, S-N. G. Chu, and A. Y. Cho, Nature (London) 358, 565 (1992)] using the transfer-matrix method. We compute the rate for intrasubband transitions from the ground state to the CBS and derive a sum rule. Finally we show how to improve the confinement of a CBS while keeping the energy fixed.
\end{abstract}

DOI: 10.1103/PhysRevB.67.085318

PACS number(s): 73.21.Cd, 78.67.De, 03.65.Ge

\section{INTRODUCTION}

In 1992, an experiment of Capasso et al. demonstrated ${ }^{1}$ the existence of a well-localized continuum bound state (CBS) in a semiconductor superlattice consisting of one thick quantum well surrounded on both sides by several GaInAs-AlInAs well/barrier layers constructed to act as $\lambda / 4$ Bragg reflectors. As suggested by Lenz and Salzman, ${ }^{2}$ the central well was made double the width of the lattice wells, to act as a $\lambda / 2$ Fabry-Perot resonator. Subsequently, Weber ${ }^{3}$ studied the existence of such states using the transfer-matrix method. Among other things, he showed that the Bragg condition need not be very well satisfied for a confined state to exist. Sung et al. ${ }^{4}$ have also studied above-threshold confined states, in a different material system, GaAs/AlGaAs. Indjin et al. ${ }^{5}$ made an exhaustive study of CBSs in systems where the unit cell is piecewise constant, with two layers. Finally, Wang et al. ${ }^{6}$ have discussed the parity sequence of subthreshold bound states localized on a defect, and the transition rates between them.

In this paper we provide further insight into the phenomenon of CBSs by relating them to surface states, whose properties were explained by Shockley in a famous paper. ${ }^{7}$ An infinite periodic system, illustrated in Fig. 1(c), allows Bloch states with the periodicity of the lattice. If the system is truncated on one side, or on both sides, [Fig. 1(b)] then one can discuss scattering states with energies above threshold, and bound states below threshold. The transfer-matrix method is well adapted to discuss such a periodic system. For convenience we will use the notation of our previous papers. 8,9

Among the bound states of a finite periodic array are Bloch-like states whose amplitude is spread more or less uniformly over the lattice, and the surface states whose densities are concentrated at the ends. The former usually occur in the allowed energy bands of the infinite lattice, while the surface states necessarily occur in the forbidden bands. Their wave functions decay exponentially outside the array and like $\pm e^{-\theta}$ from cell to cell inside, where $\theta$ is the imaginary part of the Bloch phase.

Another way to truncate an infinite lattice is to cut it in the middle and pull the two halves apart. This introduces a de- fect [Fig. 1(d)] which may be either a well or a barrier. As emphasized by Weber, ${ }^{3}$ the condition for a state localized on a defect of an infinite lattice is that the wave function at the edge of the defect matches to a decaying Bloch eigenstate of the unit cell, that is, the wave function will decay by $\pm e^{-\theta}$ from cell to cell. There are also antibound states, where the match is to the growing eigenstate. In either case, the Bloch phase must be complex, so such states exist only in the forbidden zones, in common with surface states.

In Sec. II, we briefly introduce the transfer matrix for a system with position-dependent effective mass. In Sec. III we apply it to the Capasso experiment. We determine the width of the central well to provide a CBS at a desired energy. In Sec. IV we discuss the relation between these states and states in a box, illustrated in Figs. 1(a) and 1(f). In Sec. $\mathrm{V}$ we compute the transition rate from the ground state to continuum states in the neighborhood of the CBS, and derive

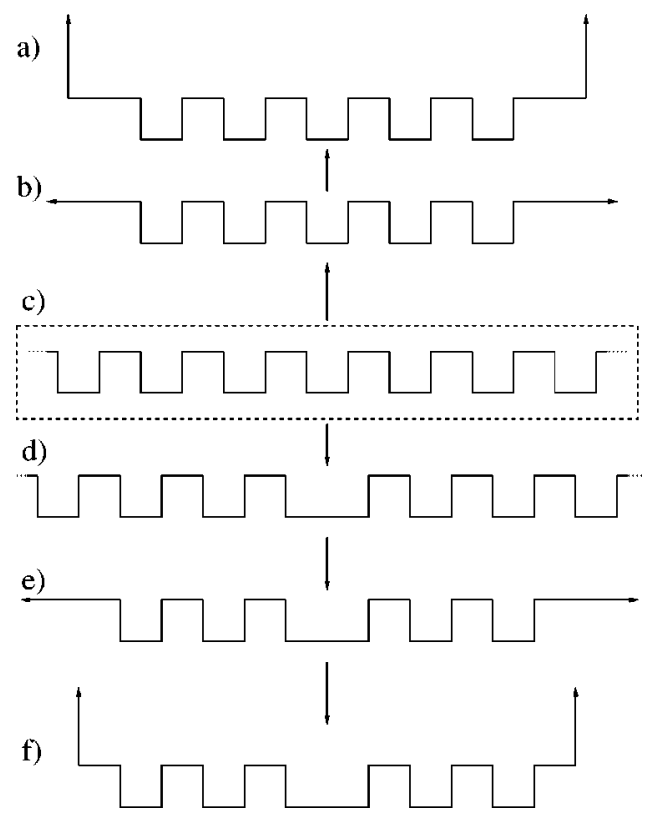

FIG. 1. Schematic drawing of (c) an infinite array, truncated to (b) a finite array, and enclosed in (a) walls; or with (d) a defect, also (e) truncated, and (f) enclosed. 
a sum rule showing that the total strength depends very little on the number of cells involved. Finally, in Sec. VI we discuss how to improve the confinement of a CBS of fixed energy, proposing an experimental arrangement to test this.

\section{TRANSFER MATRIX}

In the envelope function approximation, an electron in the conduction band is described by solutions of the Schrödinger equation with a variable effective mass

$$
-\frac{\hbar^{2}}{2} \frac{d}{d x}\left[\frac{1}{m(x)} \frac{d}{d x} \psi\right]+(V(x)-E) \psi=0 .
$$

For convenience we will discuss the situation where the entire system has reflection symmetry, such as in the specific examples discussed by Weber, Capasso, Indjin, Wang, et $a l ., 1,5,6$ Then it is sufficient to consider only $x>0$ and reflect the solutions in the origin.

We factorize $m(x)=m_{e} m^{*}(x)$ into the bare mass $m_{e}$ and the dimensionless $m^{*}$, and use $\hbar^{2} / 2 m_{e}=3.81 \mathrm{eV}^{2}$ in all calculations. In Weber's model both the potential and the effective mass are piecewise constant functions, but the method is valid even if $V(x)$ and $m^{*}(x)$ vary continuously within the potential cell.

The transfer matrix for the unit cell of width $d$ of the lattice is constructed out of two independent solutions: $v(x)$, which has value 1 , slope 0 at the left edge of the unit cell, and $w(x)$, which has value 0 and slope $m^{*}(0)$ there. It is

$$
W(d)=\left(\begin{array}{cc}
v & w \\
v^{\prime} / m^{*} & w^{\prime} / m^{*}
\end{array}\right)
$$

and satisfies

$$
\left.\left(\begin{array}{c}
\psi \\
\psi^{\prime} / m^{*}
\end{array}\right)\right|_{d}=\left.W(d)\left(\begin{array}{c}
\psi \\
\psi^{\prime} / m^{*}
\end{array}\right)\right|_{0} .
$$

Here the solutions $v, w$ without argument are evaluated at the right edge, $x=d-0$, and $\psi$ is an arbitrary solution. In a periodic system, $W(d)$ depends only on the length of the cell, not its position. Since $\psi$ and $\psi^{\prime} / m^{*}$ are continuous at interfaces, to move one interval further to the right, one simply multiplies again by the appropriate transfer matrix. Any discontinuity in the derivative is automatically taken into account.

The determinant of $W(d)$ is the Wronskian of two independent solutions $v(x), w(x)$ and is a constant. Eigenvalues of the transfer matrix satisfy

$$
\lambda^{2}-2 \cos \phi \lambda+1=0,
$$

where

$$
2 \cos \phi \equiv \operatorname{Tr} W=v+w^{\prime} / m^{*},
$$

and when the Bloch phase $\phi$ is a real angle, they are $\lambda$ $=e^{ \pm i \phi}$. Raising the energy from the potential minimum, one is in a forbidden band where $|\cos \phi|>1$. In this region of energy, $\phi=i \theta$ is imaginary. Following this, the first allowed band occurs within which $\phi$ increases from zero to $\pi$. Then in the next forbidden band, with $\cos \phi<-1, \phi=\pi+i \theta$ becomes complex. In the $p$ th forbidden band, $\phi=p \pi+i \theta$ and the eigenvalues are $\lambda=(-)^{p} e^{ \pm \theta}$.

\section{A. Surface states}

Because we have assumed reflection symmetry, states of the whole system will have either even or odd parity. Suppose that the infinite array is truncated so that there are $N$ cells to right of the origin as in Fig. 1(b). Then the condition for a bound state is that the wave function at the right edge of the array matches to a decaying solution outside (here we suppose constant potential outside, but that can be changed trivially):

$$
\left.\left(\begin{array}{c}
\psi \\
\psi^{\prime} / m^{*}
\end{array}\right)\right|_{N d}=\left.W^{N}\left(\begin{array}{c}
\psi \\
\psi^{\prime} / m^{*}
\end{array}\right)\right|_{0} .
$$

For an even bound state, $\psi=v(x)$, on the right-hand side $W^{N}$ acts on $(1,0)$ while for an odd state, $w(x)$, it acts on $(0,1)$. This gives the log derivative

$$
\left.\frac{\psi^{\prime}}{m^{*}(d) \psi}\right|_{N d}=\frac{\left(W^{N}\right)_{2 s}}{\left(W^{N}\right)_{1 s}}=\frac{-\kappa}{m_{\text {out }}^{*}},
$$

where $s=1$ (2) for even (odd) states, $m^{*}(d)$ is the effective mass inside the edge of the last cell, while $m_{\text {out }}^{*}$ is the value outside, and $E=V_{\text {out }}-\hbar^{2} \kappa^{2} /\left(2 m m_{\text {out }}^{*}\right)$. By construction, the $W$ matrix is real, so the energy $E$ must lie below the external potential $V_{\text {out }}$. On the other hand, a surface state can exist only when $E$ is in a forbidden zone, with complex $\phi=p \pi$ $+i \theta$. In such a zone, ${ }^{8}$

$$
\begin{aligned}
W(N d) & =W^{N}(d) \\
& =(-)^{(N-1) p}\left[\frac{\sinh N \theta}{\sinh \theta} W-(-)^{p} \frac{\sinh (N-1) \theta}{\sinh \theta}\right] .
\end{aligned}
$$

Equations (5) and (6) allow one to search for energies where surface states occur.

\section{B. Continuum bound states}

Suppose that the infinite periodic array is cut at the origin and an extra well of width $2 c$ is placed between the two sections, as in Fig. 1(d). Let $T(c)$ be the transfer matrix [as in Eq. (2)] that takes the wave function from the origin to $c$. Its columns are the even- and odd-parity solutions within the central well. In order for a CBS to exist, the first (or second) column of $T(c)$ must match to a decaying eigenstate of the unit cell of the semi-infinite array to the right. In other words, one of the columns of $T(c)$ must satisfy the eigenvalue equations for $W(d):^{3}$

$$
(W(d)-\lambda I)\left[\begin{array}{c}
\psi(c) \\
\psi^{\prime}(c) / m^{*}
\end{array}\right]=0,
$$




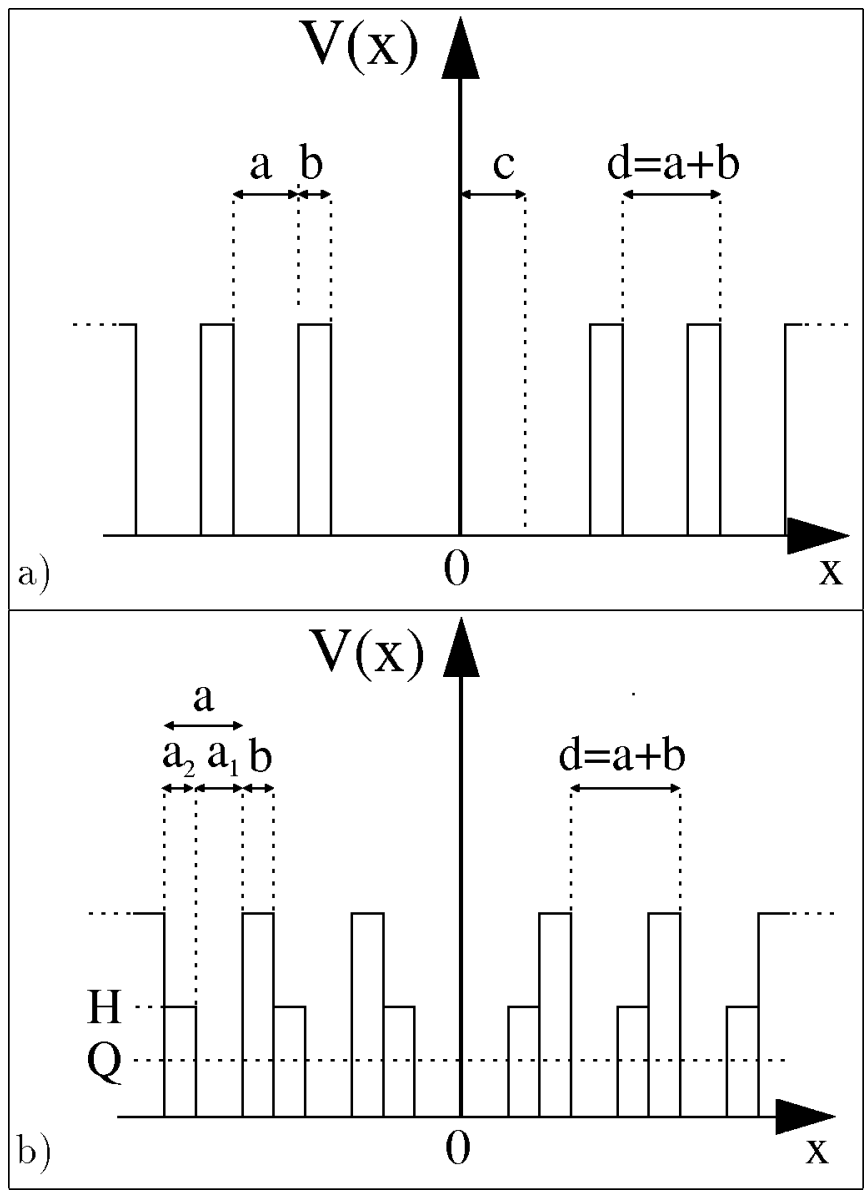

FIG. 2. A unit cell of the lattice for (a) Weber's model, and (b) the split-well configuration of Sec. VI.

$$
\frac{\psi^{\prime}(c)}{m^{*}(c) \psi(c)}=\frac{\lambda-W_{11}(d)}{W_{12}(d)}=\frac{W_{21}(d)}{\lambda-W_{22}(d)} .
$$

Either of these equations can be used to search for CBSs. If they are satisfied with real $\lambda$ being the smaller $\lambda_{-}$(larger $\lambda_{+}$) eigenvalue, then a CBS [or an antibound state (ABS)] exists at that energy. The only difference between them and surface states is the numerical value of the boundary condition that has to be satisfied where the lattice meets the defect or the surface.

\section{CAPASSO-WEBER EXAMPLE}

The array constructed by Capasso can be modeled as a sequence of potential wells of width $w=16 \AA$, depth $V_{w}$ $=0$, and barriers of width $b=39 \AA$ and height $V_{b}$ $=500 \mathrm{meV}$. The energy-dependent effective mass in each layer is given by ${ }^{10,11}$

$$
\begin{aligned}
& m_{w}^{*}=0.043\left[1+\left(E-V_{w}\right) / E_{w}\right], \\
& m_{b}^{*}=0.073\left[1+\left(E-V_{b}\right) / E_{b}\right],
\end{aligned}
$$

where $E_{w}=0.88 \mathrm{eV}$, and $E_{b}=1.49 \mathrm{eV}$ are the effective band gaps of InGaAs well and AlInAs barrier materials.

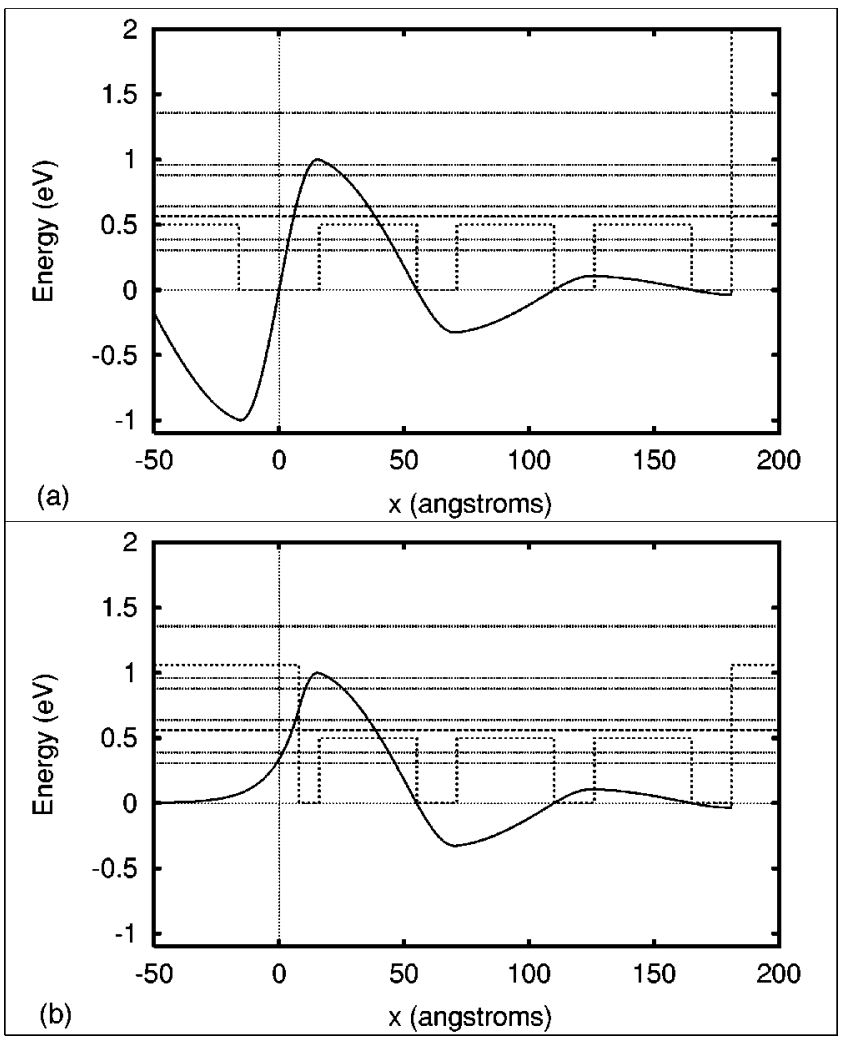

FIG. 3. Wave functions of (a) a CBS and (b) a surface state compared.

In this example, the potential is piecewise constant, so the transfer matrix can be constructed from factors of the type

$$
T(c)=\left[\begin{array}{cc}
\cos c k_{c} & \left(m_{c}^{*} / k_{c}\right) \sin c k_{c} \\
-\left(k_{c} / m_{c}^{*}\right) \sin c k_{c} & \cos c k_{c}
\end{array}\right],
$$

where $k_{c}^{2}=2 m_{e} m_{c}^{*}\left(E-V_{c}\right) / \hbar^{2}$ is the wave number inside the layer and $m_{c}^{*}$ is the effective mass there.

We follow Weber in taking the unit cell to be a well followed by a barrier, as illustrated in Fig. 2(a). Then

$$
W(d=w+b)=T(b) T(w) .
$$

The situation of a central defect being a double width well, corresponds to $c=0$. Indjin et al..$^{5}$ have written out the forms in all detail.

The transfer matrix from the origin to $c+N d$ is $W^{N}(d) T(c)$. For any $c>-w / 2$ the central well constitutes a defect in the superlattice. If $N \rightarrow \infty$, the argument of Sec. II B applies, and the wave function will only be localized near the origin when one of the columns of $T(c)$ is an eigenvector of $W(d)$ with the decaying eigenvalue $\pm e^{-\theta}$. The left-hand side of Eq. (8) [cf. Eq. (10)] is either $-k_{w} \tan k_{w} c / m_{w}^{*}$ (for an even state) or $k_{w} \cot k_{w} c / m_{w}^{*}$ (odd state). But the identity $\cot (x+\pi / 2)=-\tan (x)$ means that the solutions for odd states can be found simply by adding $\pi / 2$ to the value of $k_{w} c$ of an even state solution. Given any solution, another one, which differs only by the number of nodes in the central 


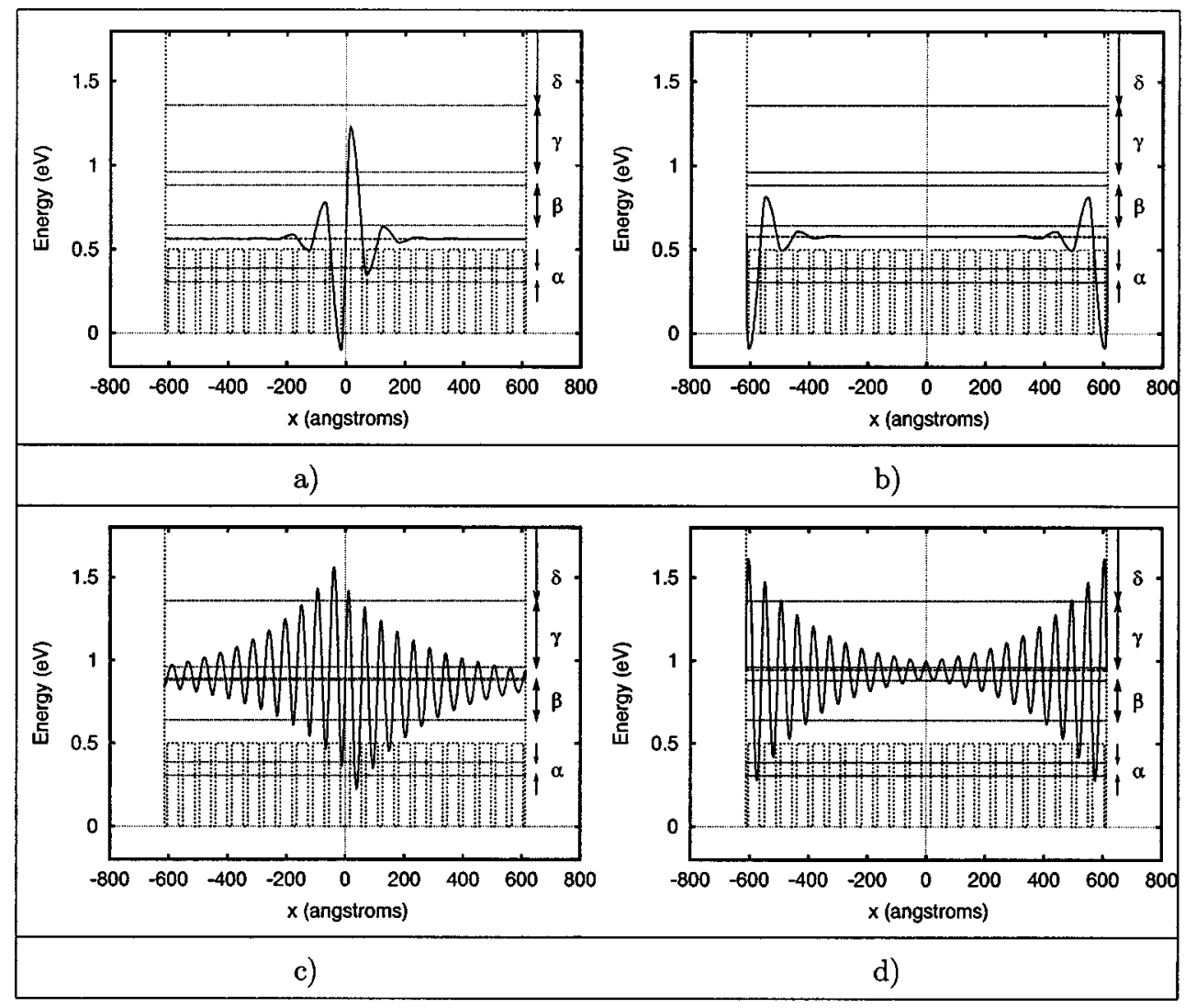

FIG. 4. States in a box, for $c=8 \AA$, also showing (a) the CBS at $563 \mathrm{meV}$, (b) the ABS at $577 \mathrm{meV}$, (c) the CBS at $891 \mathrm{meV}$, and (d) the ABS at $946 \mathrm{meV}$. At right, $\alpha$ labels the first allowed band from $307 \rightarrow 387 \mathrm{meV}, \beta$ the second allowed band from $641 \rightarrow 881 \mathrm{meV}, \gamma$ the third allowed band from $960 \rightarrow 1357 \mathrm{meV}$, and $\delta$ the fourth allowed band from $1357 \mathrm{meV}$. Dotted lines show the potential cells, in eV; wave functions are dimensionless and are drawn with base lines at the energy eigenvalue.

well, can be obtained by adding $\pi / k_{w}$ to $c$. Hence, it is the differences in width of the central well that go by half wavelengths, not the whole width.

To illustrate the close relationship between CBS and surface states, in Fig. 3 we show in the upper panel the right side of a six-cell lattice with the central well of width $2 w$, having the CBS at $563 \mathrm{meV}$. In the lower part we show a three-cell lattice with a surface state at the left edge decaying into the potential barrier, chosen so the slope is exactly the same for both wave functions. The only difference is that one state passes through zero at the origin to make an odd-parity wave function, while the other decays exponentially; within the lattice they are identical.

Weber $^{3}$ noted that a well-confined CBS was obtained even when the widths $w$ and $b$ of the well and barrier were rather far from the optimal values. Indjin et $a .^{5}$ made an exhaustive study of the location of the CBS as $w, b$, and $c$ were varied, so we will only make one comment. If both $w$ and $b$ are varied while keeping the energy of the CBS fixed, the minimum value of $\left|\lambda_{-}\right|$is obtained with both $w k_{w}$ and $b k_{b}$ $=\pi / 2$. This should be expected, because once we fix the energy of the state, the effective masses are also fixed. Then the optimization of $w k_{w}$ and $b k_{b}$ proceeds exactly as for an energy-independent Kronig-Penney potential, for which the Bragg condition is optimal, as one can easily show analytically.

\section{RELATION TO STATES IN A BOX}

Kalotas and Lee ${ }^{12}$ considered the states obtained by enclosing a finite number of cells between infinite walls. [See Figs. 1(a) and 1(f) for illustrations.] This discretizes the continuum, so all states become discrete. Well-localized states that decay quickly enough will be scarcely affected by the walls. States spread over the whole lattice will become a discrete set maintaining similar character. An ABS whose magnitude grows away from the origin will be squeezed against the walls of the box.

Figure 4 shows an example where we have taken $N=11$ cells on each side of the origin. Figure 4(a) can be compared with the CBS at $E=563 \mathrm{meV}$ of the $c=0$ example of $\mathrm{Ca}-$ passo and Weber. Even with just three cells on either side, the state is hardly shifted from its position in the infinite array. Figure 4(c) shows the second CBS at $891 \mathrm{meV}$, in the next higher forbidden miniband. An ABS occurs between each pair of CBSs; two examples are shown in Figs. 4(b) and 4(d). If the box walls were taken away the ABS wave functions would grow without limit, so they are not normalizable states and are of only theoretical interest.

In Fig. 5 we show the spectrum of box states as a function of $N$, again for the $c=0$ central well case. The energies of the single-cell states change little as more cells are added. The new states that appear fill up the allowed bands. To 


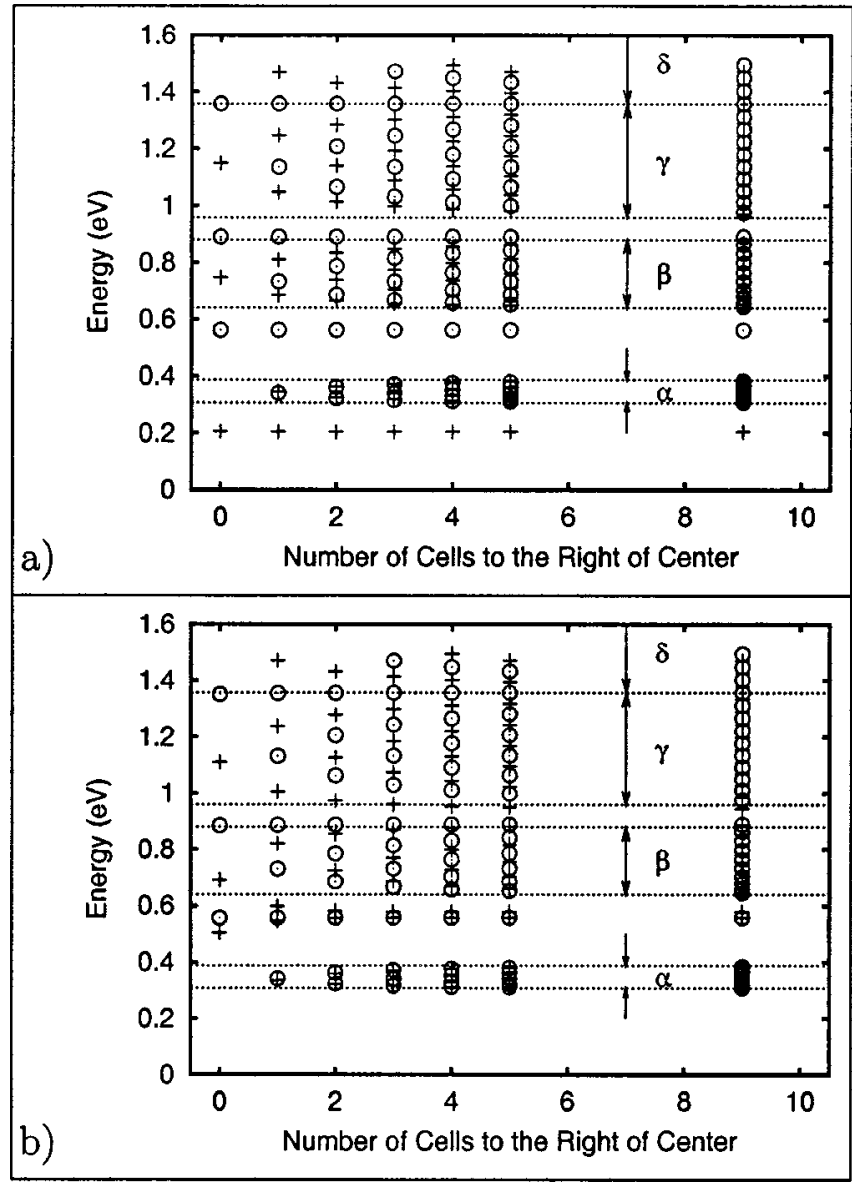

FIG. 5. Even (crosses) and odd (circles) state energies (in a box) versus number $N$ of cells to the right of the central defect. (a) is for a central well, and (b) for a central barrier. Bands are labeled as in Fig. 4.

understand this, it is convenient to consider a system with a hard wall at the origin, then $N$ identical cells, followed by a hard wall at the right. The allowed wave functions are those that vanish at the origin (odd-parity states of the symmetric system), and the hard-wall boundary condition at the right edge is $\psi(x=N d)=0$. In view of Eq. (4), this requires that the element $\left(W^{N}\right)_{12}=0$. Since in an allowed band

$$
W^{N}(d)=\frac{\sin N \phi}{\sin \phi} W(d)-\frac{\sin (N-1) \phi}{\sin \phi} I,
$$

this can be written

$$
\left(W^{N}\right)_{12}=0=\frac{\sin N \phi}{\sin \phi} W_{12}(d) .
$$

This shows that bound states can occur in either of two ways. First, they can occur as single-cell bound states, where the second factor vanishes. These states have $N$ nodes, and the wave function vanishes at every cell boundary. Alternatively, the combinatorial factor $\sin N \phi / \sin \phi$ may vanish, and in an allowed band there are $N-1$ such states with $N \phi=m \pi, m$ $=1,2, \ldots, N-1$. The single-cell state may occur in a forbidden zone, but the others can only occur for real $\phi$, in an allowed band. For nonzero $c$ one has to multiply $W^{N}(d)$ from the right by the additional transfer matrix $T(c)$ for the central well, and then the simple factorization won't be exact. In practice the states remain in the allowed band all the same.

Figure 5 provides another example of the similarity of CBSs and surface states. In Fig. 5(a), the CBS lies in the middle of the first forbidden zone, while in the lower panel there are two such states, one derived from the allowed band $\alpha$ and the other from band $\beta$.

Incidentally, Fig. 5 provides an explanation for the conundrum of Wang et al. ${ }^{6}$ They were concerned as to what happens to bound states which exist in some potential, when it is cladded on each side by an infinite superlattice. As is clear from Fig. 5(a), these states continue to exist at the same energy. The third state, in band $\beta$, alternates in parity depending on its order in the ladder of states as additional potential wells are added on each side, but the energy scarcely changes. Each of these states is a linear combination of the original state and the new ones from the side cells that lie close in energy. The same situation holds for the fifth state, in band $\gamma$. Of course, as the lattice becomes wider the states in an allowed band will spread out over the entire width. The difficulty of Wang et al. was that they jumped from no lattice to the entire system, and examined only states in the forbidden bands.

\section{TRANSITION RATES}

Introducing the vector potential into the Hamiltonian, Eq. (1), leads to the excitation operator

$$
\frac{e A}{2 c}\left[p \frac{1}{m(x)}+\frac{1}{m(x)} p\right] \equiv \frac{-i \hbar e A}{2 m_{e} c} S
$$

with dimensions of energy. In defining the operator $S$ (dimensions of inverse length), we have factored out the bare electron mass, leaving only the dimensionless effective mass $\left(m^{*} \sim 0.06\right)$ inside. The vector potential $A$ is assumed to be a function of $x$, so it commutes with the mass. By invoking the Coulomb gauge we make it commute with the momentum as well.

According to the Golden rule, the transition rate is

$$
w_{i f}=\frac{2 \pi}{\hbar}\left(\frac{e A \hbar}{2 m_{e} c}\right)^{2}\left|\left\langle\Psi_{f}|S| \Psi_{i}\right\rangle\right|^{2} \rho\left(E_{f}\right),
$$

where $\rho\left(E_{f}\right)$ is the density of final states. The factors before the matrix element have dimensions of length squared times energy per second, and these are omitted from our calculations. The matrix element squared times the density of states is therefore (energy length-squared) ${ }^{-1}$, and this is what we plot in Figs. 6 and 9. After integrating over energy, we use units of $\AA^{-2}$ for the total strength.

In the Capasso experiment, the ground state has even parity, so its derivative is odd, and transitions are allowed only to odd-parity excited states. Also the lattice is finite rather than infinite, so the transitions are to states in the continuum. In the neighborhood of the CBS, the continuum wave func- 
tion has a large normalization inside the central well, and this causes the transition rate to peak at or near this energy.

We again consider the case of a central well of half width $c$ surrounded by $N$ additional cells on each side. The oddparity excited state with Dirac delta-function normalization has wave function

$$
\begin{aligned}
\Psi_{f}(x) & =B_{0} \sin \left(k_{w} x\right), \quad|x|<c \\
& =\frac{1}{\sqrt{\pi}} \sin \left(k_{b} x+\delta\right), \quad x>c+N d,
\end{aligned}
$$

where $E-V_{b}=\hbar^{2} k_{b}^{2} /\left(2 m m_{b}^{*}\right)$ measures the energy above the top of the barrier in the asymptotic zone, and $k_{w}^{2}$ $=2 m m_{w}^{*} E / \hbar^{2}$ is the wave number inside the central well. Using the transfer matrix $W(d)$ to cross $N$ cells gives

$$
\frac{1}{\sqrt{\pi}}\left(\begin{array}{c}
\sin \left[k_{b}(c+N d)+\delta\right] \\
\nu_{b} \cos \left[k_{b}(c+N d)+\delta\right]
\end{array}\right)=B_{0} W^{(N)}\left(\begin{array}{c}
\sin k_{w} c \\
\nu_{w} \cos k_{w} c
\end{array}\right),
$$

where $\nu_{w}=k_{w} / m_{w}^{*}, \nu_{b}=k_{b} / m_{b}$, and $W^{(N)}=W^{N}(d)$, so that

$$
\frac{1}{\nu_{b}} \tan \left[k_{b}(c+N d)+\delta\right]=\frac{W_{11}^{(N)} \sin k_{w} c+W_{12}^{(N)} \nu_{w} \cos k_{w} c}{W_{21}^{(N)} \sin k_{w} c+W_{22}^{(N)} \nu_{w} \cos k_{w} c}
$$

determines $\delta$, and

$$
\begin{aligned}
& \frac{1}{\sqrt{\pi}} \sin \left[k_{b}(c+N d)+\delta\right] \\
& \quad=B_{0}\left[W_{11}^{(N)} \sin k_{w} c+W_{12}^{(N)} \nu_{w} \cos k_{w} c\right]
\end{aligned}
$$

gives the normalization $B_{0}$. Note that the matrix elements of $W^{(N)}$ can be easily computed from those of $W(d)$ using Eq. (6) [or Eq. (12) when the Bloch phase $\phi$ is real]. One need not solve explicitly for the phase shift $\delta(E)$ because only $\left|B_{0}\right|^{2}$ is required to compute the transition rate, and the identity $\sin ^{2} z=\tan ^{2} z /\left(1+\tan ^{2} z\right)$ can be used in Eq. (19).

With the above equations we can construct the wave function $\Psi_{f}(x)$ as follows. Wave functions $v(x), w(x)$ in a unit cell of the lattice are defined in Eq. (2). Within the $r$ th cell following $x=c,(r=1,2, \ldots)$ the wave function $\Psi_{f}(x)$ is written as

$$
\Psi_{r}(x)=A_{r} v(x-c-r d+d)+B_{r} w(x-c-r d+d)
$$

and from the matching at $x=c$ we have $A_{1}=B_{0} \sin k_{w} c, B_{1}$ $=B_{0} \nu_{w} / \nu_{b} \cos k_{w} c$. In general,

$$
\left(\begin{array}{c}
A_{r+1} \\
B_{r+1} / m^{*}
\end{array}\right)=W(d)\left(\begin{array}{c}
A_{r} \\
B_{r} / m^{*}
\end{array}\right), r=1, \ldots,(N-1) .
$$

Knowing $\Psi_{r}(x)$ in each cell allows the calculation of the matrix element in Eq. (15).

The ground-state wave function is computed in a similar manner. Inside the central well it is

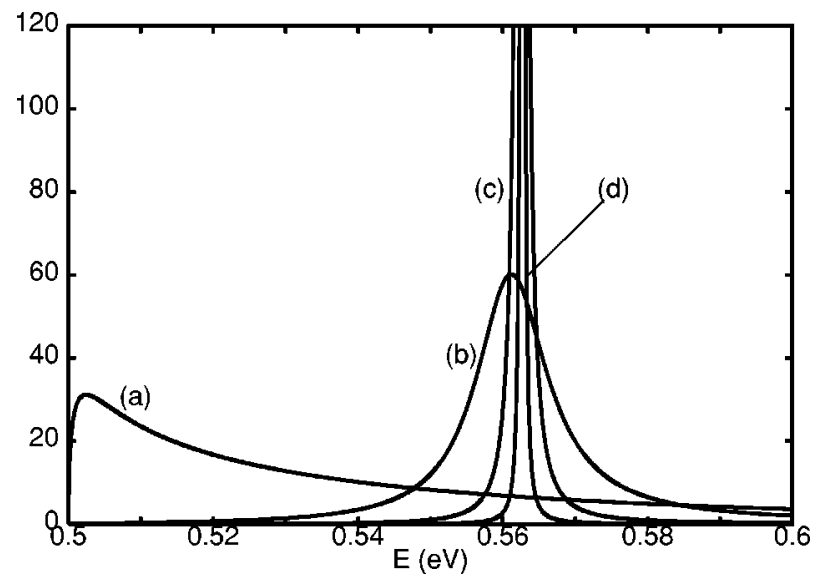

FIG. 6. The transition strength times the density of states (units $\mathrm{eV}^{-1} \AA^{-2}$ ) for (a) a central well of width $32 \AA$, and (b) a central well surrounded by one cell, (c) two cells, and (d) three cells on each side.

$$
\Psi_{0}(x)=N_{0} \cos \left(k_{w} x\right), \quad|x|<c
$$

and at $x=c+N d$ it must match to a decaying exponential, as in Eq. (5). If the state is well bound it is a good approximation to use the Kalotas state which vanishes at the edge of the lattice or the Weber state that, in principle, extends to infinity. The normalization constant $N_{0}$ must be computed by summing the normalization integrals from every cell as well as from the central well. If one integrates $\langle v \mid v\rangle,\langle v \mid w\rangle$, and $\langle w \mid w\rangle$ over the unit cell, then it is just a matter of multiplying these integrals by the coefficients in the $r$ th cell and summing.

Because the effective mass depends both on position and energy (and therefore momentum), it is not obvious how to evaluate the matrix elements of the transition operator $S$. It is reasonable, in the term $p / m^{*}$, to let $p$ act on the excited state $\psi_{E}$ and interpret the effective mass as being at that energy. Conversely, in the $\left(1 / m^{*}\right) p$ term, where $p$ acts on the initial state, we use the ground-state effective mass $m_{0}^{*}$. Then,

$$
\left\langle\psi_{E}|S| \psi_{0}\right\rangle=\int \psi_{E} \frac{1}{m^{*}\left(E_{0}\right)} \frac{d \psi_{0}}{d x} d x-\int \frac{d \psi_{E}}{d x} \frac{1}{m^{*}(E)} \psi_{0} d x
$$

Since $\psi^{\prime} / m^{*}$ is continuous at interfaces between wells and barriers, the integrand is continuous, despite the jumps in $m^{*}$. When the effective mass $m^{*}$ is piecewise constant, we can evaluate the integral over a series of intervals of constant $m^{*}$ [here interpreted as $\left.m^{*}(x, E)\right]$.

The squared matrix element, including the density-ofstates factor, is plotted as a function of energy in Fig. 6, which is to be compared with Fig. 2 of Capasso et al. ${ }^{1}$ (However, their figure has normalized the peak height to unity in each case, obscuring the fact that the integrated strength is constant.) As the number of side wells increases, the computed excitation function rapidly becomes very narrow. It shows that even a small number of cells is sufficient to give a well-confined state. We also find increasing strength in the second allowed band near $700 \mathrm{meV}$ as cells are added. The 


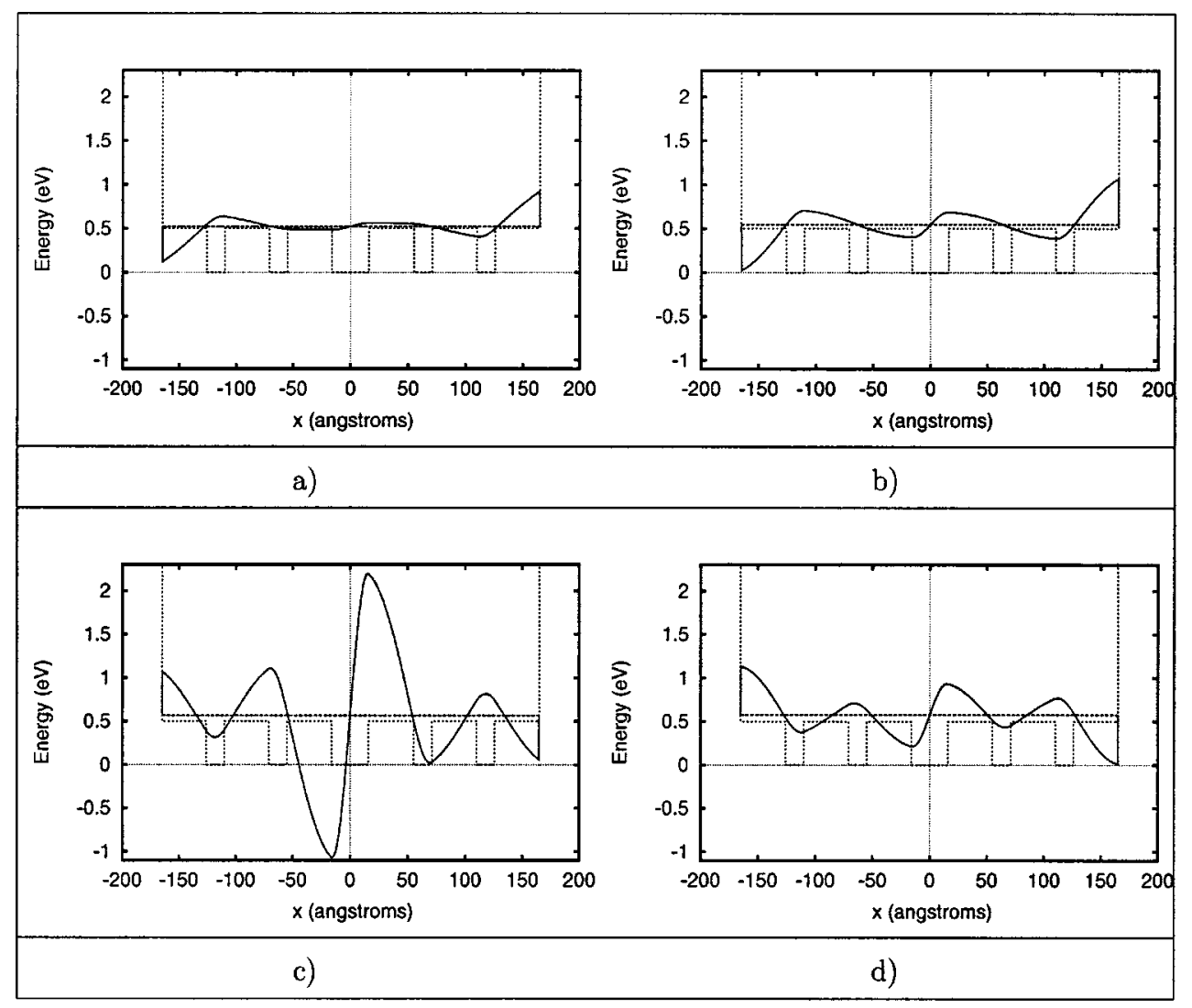

FIG. 7. Four representative continuum wave functions, for $c=8 \AA$, at energies (a) $E=521 \mathrm{meV}$, (b) $545 \mathrm{meV}$, (c) $565 \mathrm{meV}$, and (d) 573 $\mathrm{meV}$. Dotted lines show the potential cells, in $\mathrm{eV}$; wave functions are dimensionless and are drawn with base lines at the corresponding energy.

integrated strength under the main peak (from 500 to 640 $\mathrm{meV}$ ) varies by only a few percent.

To illustrate how the continuum wave functions evolve in the region of the CBS, we show in Fig. 7 four cases spanning the energy range. We match the wave function after $N=3$ cells; see Eq. (17). It can be seen that as one passes over the CBS energy at $563 \mathrm{meV}$, an additional node appears in the wave function on each side of the origin. Away from this resonance, [Figs. 7(a) and 7(b)] the wave function consists mainly of the growing solution in the lattice, so the amplitude is largest at the outside edge [where it is fixed, according to Eq. (16)]. Close to the CBS [Fig. 7(c)], there is a large component of the decaying solution, making the amplitude in the central well large. Increasing the energy again [Fig. 7(d)] brings back more of the growing solution. At $577 \mathrm{meV}$, the position of the ABS, only the growing solution would contribute. If we had more than $N=3$ cells, the effects would be even more pronounced.

While the peak in the transition strength becomes very narrow as the number of lattice cells $N$ increases from zero to 3 , the integrated strength is almost constant. This can be understood from the sum rule which follows from Eq. (15), and is discussed in Appendix A. The total strength, $M_{2}$, is defined in Eq. (A2). In addition to the integral over the continuum, when there are $N$ Bragg reflectors on either side of the central defect, there will be $N$ discrete odd-parity bound states, which must also be included in the sum. Typically these account for something like $6 \%$ of the total strength. These odd bound states are shown in Fig. 8, for the case $N$ $=2$. In this figure, the wave functions are remarkably similar inside the region where the ground state is large, so they contribute almost equally to the sum rule.

Turning now to the results, one has to distinguish between

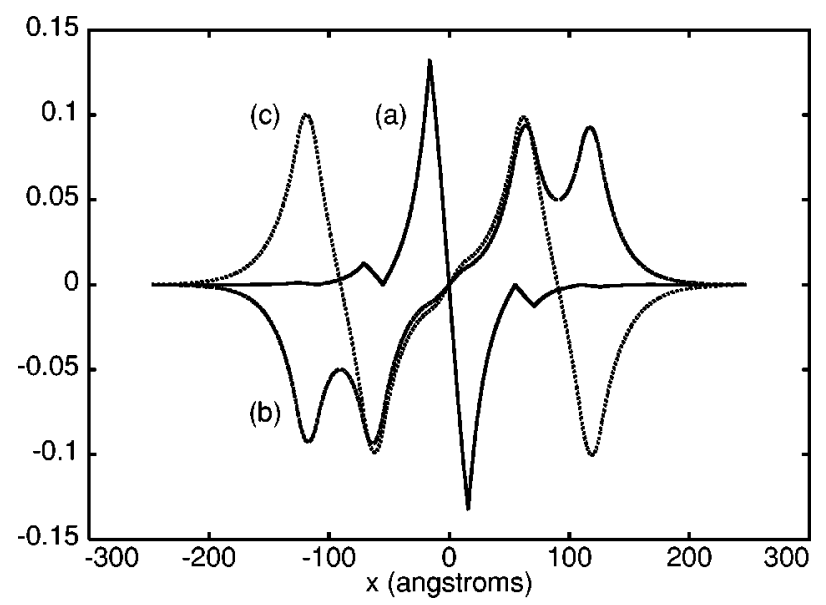

FIG. 8. The derivative of the (a) ground-state wave function (over $m^{*}$ ) and (b) and (c) the first and second odd-parity excited wave functions, for a five well potential (two identical cells on each side of a central well), enclosed in a box, illustrating the similar overlap near the origin. 
the no-reflector case and the $N$-reflector case. In the former, the integrated transition strength (ITS), $M_{2}$, is about $1.4 \AA^{-2}$. The strength is very broadly distributed above threshold; see Fig. 6(a). We have looked at $N=1-5$ cells on each side. For these cases, the ITS is around $1.35 \AA^{-2}$, of which about 0.09 comes from the bound odd-parity excited states. As $N$ increases, the ITS fluctuates only a little. The strength remains highly concentrated into the CBS peak (about $80 \%$ ), the remainder being spread quite widely (6\% in the bound states and $14 \%$ in the continuum). Because the CBS peak becomes so narrow, we estimate the integral under it by assuming a Breit-Wigner shape, and deducing the height and width from the calculations.

The sum rule, calculated according to Eq. (A3), is always about $6 \%$ to $10 \%$ higher than the ITS, if we (arbitrarily) set the doorway state mass $m_{E}$ to be at $E=E_{C B S}=563 \mathrm{meV}$. The main term in Eq. (A3) is proportional to $\left(1 / m_{0}\right.$ $\left.+1 / m_{E}\right)^{2}$, so we can easily adjust the doorway energy to ensure that the sum rule will agree with the ITS. We call this the effective doorway energy $E_{D}$. With no reflectors $E_{D}$ is about $740 \mathrm{meV}$ (535 above the ground-state energy). This is reasonable since the excitation strength is very broadly distributed above threshold. With one reflector (on each side), $E_{D}$ drops to $660 \mathrm{meV}$, but then it slowly rises, at least up to $N=5$, where it reaches $670 \mathrm{meV}$.

\section{OPTIMAL CBS CONFINEMENT}

In this section we discuss general principles for designing a CBS with a narrow distribution of transition strength. Consider a general unit cell of width $d$, within which the potential and the effective mass are arbitrary functions of $x$. (Since we will fix the CBS energy, this allows for energy dependence of the effective mass.) Now let us arbitrarily divide the cell into two parts so that widths $a$ and $b$ add to $d$, and we denote by $W^{a}, W^{b}$ the transfer matrices of the two parts. When $a$ is on the left, we have $W^{d}=W^{b} W^{a}$. If the whole array is symmetric about the origin, there will be two type- $a$ portions together at the origin, and the sequence of potentials is $\ldots b a b a \ldots b a \mid a b \ldots a b a b \ldots$ (This is a special case corresponding to $c=0$.) When we look for odd-parity confined states of such an array, it is equivalent to putting a hard wall at the origin, and solving only the right side. The wave function at the edge of the first cell will be, using Eq. (2),

$$
\left.\left(\begin{array}{c}
\psi \\
\psi^{\prime} / m^{*}
\end{array}\right)\right|_{d}=\left(\begin{array}{c}
W_{12}^{d} \\
W_{22}^{d}
\end{array}\right)=\lambda\left(\begin{array}{l}
0 \\
1
\end{array}\right) .
$$

The second equality holds if we imagine an infinite array, and demand an eigenstate with the wave function in each cell differing only by a factor $\lambda$. This wave function will vanish at both $x=0$ and $d$, and in the second cell, the value of $\psi^{\prime} / m^{*}$ will differ by a factor $\lambda$ from the first. The condition that must be satisfied is $W_{12}^{d}=0$, and then $\lambda=W_{22}^{d}$. One can then show (see Appendix B) that the eigenvalue is

$$
\lambda=-\frac{W_{12}^{a}}{W_{12}^{b}}=-\frac{w_{a}(a)}{w_{b}(b)} .
$$

What this tells us is that to make the eigenvalue as small as possible, we must make the $w_{a}(x)$ solution as small as possible at the right edge of the $a$ part cell, and conversely, $w_{b}(x)$ as large as possible at the right edge of the $b$ part cell.

The above is true for any division of the cell into two parts. In the system studied by Capasso et al., the logical division is into the two layers of GaInAs and AlInAs. In that situation, the off-diagonal elements have the form $W_{1,2}^{c}=$ $-\sin k_{c} c / \nu_{c}$, where $\nu_{c}=k_{c} / m_{c}^{*}(c=a, b)$ is the velocity. If the Bragg reflection condition holds, then $\sin k_{c} c= \pm 1$, and $\lambda=-\nu_{b} / \nu_{a}$ is just the ratio of velocities in the two parts of the cell. [This is analogous to the problem of waves on a string, with one part thin and the other thick. At the join, the displacement $y(x)$ is continuous, and the ratio of the slopes $y^{\prime}(L) / y^{\prime}(R)$ is the ratio of the velocities squared.] One sees that in the $a$ cell, the solution $w_{a}(x)$ rises to the value $w_{a}(a)=1 / \nu_{a}$, while starting from $d$ and moving backwards through the barrier region, the corresponding solution falls to the value $w_{b}(-b)=-w_{b}(b)=-1 / \nu_{b}$. Normalizing the $b$ solution to ensure continuity at $x=a$ requires the factor $\lambda$.

The result, Eq. (25), is quite surprising because in the general situation where the potential and effective mass vary arbitrarily, the dividing line can be placed anywhere. To make the eigenvalue small, one must make $w(x)$ as large as possible throughout the second part cell and as small as possible in the first. As observed by Weber, the first aspect can be achieved by choosing an energy just above the barrier $\left(\right.$ small $k_{b}$ ). To meet the Bragg condition, this forces a large $b$, and the linear variation of $w_{b}(x)$ over the barrier leads to a large wave function at $x=b$. That is why lowering the energy of the CBS in general improves confinement.

However, our aim is to improve confinement while keeping the energy of the CBS fixed. We split the $a$ part cell into two sections, $a_{1}, a_{2}$, making the left side more attractive, and the right side less so $\left(V_{w 1}<V_{w}<V_{w 2}\right)$. This is illustrated in Fig. 2(b). The greater curvature of $w_{a}(x)$ near the origin, balanced by less curvature to the right, will lead to a smaller value of $w_{a}(a)$, even if the average attraction is the same. We leave the $b$ cell fixed, but a similar strategy with less repulsion on the right side can obviously be employed there. A very complete study of surface states (but not CBSs) generated by a three-layer unit cell has been made by $\mathrm{Ku}$ charczyk ${ }^{*} \mathrm{r}$ et al. ${ }^{14}$ Of relevance here, their Fig. 4(a) shows that the strategy we developed above yields much better localization than an alternative one shown in their Fig. 4(b).

To illustrate our method, we have selected a set of heterostructures based on quaternary alloys $\mathrm{Ga}_{x} \mathrm{In}_{1-x} \mathrm{As}_{y} \mathrm{P}_{1-y}$, lattice matched to $\operatorname{InP}(x=0.468 y)$. We took information from Figs. 1.17 (for band gaps) and 1.20 (for effective masses) of Swaminathan and Macrander. ${ }^{15}$ For the band offsets, Adachi ${ }^{16}$ gives

$$
\begin{gathered}
\Delta E_{c}=268 y+3 y^{2}, \\
\Delta E_{v}=502 y-152 y^{2} .
\end{gathered}
$$


TABLE I. Optimized three-layer potentials indicating the changes in the widths $a_{1}, a_{2}, w(a)$, and $\nu_{a}$ $=k_{a} / m_{a}^{*}$, and eigenvalue $\lambda$. In all cases $b=44.2851450 \AA, \quad w_{b}(b)=2.1450935$, and $E_{C B S}$ $=563.0 \mathrm{meV}$.

\begin{tabular}{cccccc}
\hline \hline Case & $a_{1}$ & $a_{2}$ & $w(a)$ & $\nu_{a}$ & $\lambda$ \\
\hline$(Q, Q)$ & 7.9109 & 7.9109 & 0.8636 & 1.1579 & -0.4026 \\
$(0, H)_{1}$ & 9.3535 & 9.3535 & 0.8495 & 0.9092 & -0.3960 \\
$(0, H)_{2}$ & 10.4995 & 7.9109 & 0.8031 & 0.9092 & -0.3744 \\
\hline \hline
\end{tabular}

The band alignments are also discussed on p. 87 of Davies. ${ }^{17}$ Putting together this information, we arrived at the following set of parameters:

$$
\begin{aligned}
& y=1.0, m_{0}^{*}=0.043, \bar{E}_{g}=0.880 \mathrm{eV}, \\
& y=0.5, \quad m_{0}^{*}=0.061, \quad \bar{E}_{g}=1.080 \mathrm{eV}, \\
& y=0.0, \quad m_{0}^{*}=0.081, \quad \bar{E}_{g}=1.360 \mathrm{eV},
\end{aligned}
$$

where $\bar{E}_{g}$ are effective band gaps in the sense of Nelson $e t$ al..$^{10}$

In this way we can have conduction-band potential steps of $125 \mathrm{meV}$ [ from $\mathrm{Ga}_{.47} \mathrm{In}_{.53} \mathrm{As}$ to $\mathrm{Ga}_{.23} \mathrm{In}_{.77} \mathrm{As}_{.5} \mathrm{P}_{.5}$ or 250 meV to $\operatorname{InP}(y=0)]$. These are a quarter (denoted $Q$ ) or a half (denoted $H$ ) step up to the $500-\mathrm{meV}$ barrier of $\mathrm{Al}_{.48} \mathrm{In}_{.52} \mathrm{As}$.

As our baseline [denoted $(Q, Q)$ below], we take the $a$ well to consist entirely of $Q(y=0.5)$ material, so the potential floor at $125 \mathrm{meV}$ is $375-\mathrm{meV}$ below the barrier. The barrier width was fixed at $44.3 \AA$, which satisfies the Bragg condition. For a width $a=15.82 \AA$, the CBS is at $63 \mathrm{meV}$ above the top of the barrier, as in the original experiment. The eigenvalue $\lambda=-0.4026$ is not as favorable as in the original work because the well is not so deep. (Capasso et al. evidently selected the materials to have the greatest possible well-barrier potential difference.) The potential properties are summarized in Table I, top line.

Next we divide the $a$ well into two parts, one of GaInAs $(y=1)$ and the other of InP. We adjusted the widths $a_{1}$ and $a_{2}$ to keep $E_{C B S}$ fixed. In the second line of Table I, denoted $(0, H)_{1}, \quad a_{1}=a_{2}=9.353 \AA$, and the eigenvalue is $\lambda=$ -0.396 . In the third line, denoted $(0, H)_{2}$, the deeper well has width $a_{1}=10.50 \AA$, and the shallower part $a_{2}$ $=7.911 \AA$, giving $\lambda=-0.374$. This may seem a small gain, but we shall see that the improvement is significant.
We then compute the CBS properties for a finite array based on the above materials, with results shown in Tables II and III and Fig. 9, where $N$ is the number of Bragg reflectors placed on each side of the central defect. The transition strength is significantly narrower and more strongly peaked for the split-well examples. The strength to the bound states in the split-well cases is only $25 \%-30 \%$ of that of the (Q,Q) reference case, and the strength to the continuum states is much larger.

The differences in the total strength are reflected in the portion concentrated in the CBS peak. The decay constant strongly influences both the sharpness of, and the area under, the peak. Although the eigenvalue $\lambda$ (last column of Table I) differs by only a few percent among the three cases, there is a dramatic increase in peak height and decrease in peak width shown in the first six columns of Table III. This shows that the decay constant by itself is not a direct indicator of the concentration of transition strength.

Reducing the decay constant even a little has a significant effect on both the total strength, and its continuum and bound-state contributions individually. A lower value of the decay constant (in magnitude) results in better confinement of the CBS as is evidenced by the width of the peak in the transition strength curve. We conclude that the split-well strategy can produce much better confinement of the CBS. It should be feasible to confirm this method of improving the confinement of CBSs, experimentally.

\section{CONCLUSION}

We have shown that continuum bound states are closely related to surface states, because both arise as a result of perturbing an infinite periodic system. The results of Weber ${ }^{3}$ concerning the experiment of Capasso et al. were verified. In addition, by enclosing a finite array in a box, we have traced the evolution of the Bloch states in the allowed bands as the number of Bragg layers is increased.

The experimental measure of confinement is not the ei-

TABLE II. Evolution of the transition strength $\left(\AA^{-2}\right)$ with increasing number of Bragg reflectors, $N$.

\begin{tabular}{cccccccccc}
\hline \hline & \multicolumn{3}{c}{ Bound } & \multicolumn{3}{c}{ Continuum } & \multicolumn{3}{c}{ Total strength } \\
$N$ & $(Q, Q)$ & $(0, H)_{1}$ & $(0, H)_{2}$ & $(Q, Q)$ & $(0, H)_{1}$ & $(0, H)_{2}$ & $(Q, Q)$ & $(0, H)_{1}$ & $(0, H)_{2}$ \\
\hline 0 & 0 & 0 & 0 & 0.912 & 1.279 & 1.324 & 0.912 & 1.279 & 1.324 \\
1 & 0.108 & 0.0267 & 0.0319 & 0.762 & 1.188 & 1.234 & 0.870 & 1.215 & 1.266 \\
2 & 0.117 & 0.0281 & 0.0334 & 0.759 & 1.188 & 1.233 & 0.876 & 1.216 & 1.267 \\
3 & 0.118 & 0.0282 & 0.0335 & 0.754 & 1.183 & 1.229 & 0.872 & 1.211 & 1.263 \\
\hline \hline
\end{tabular}


TABLE III. Total strength under the CBS peak: dependence on number of Bragg reflectors, $N$.

\begin{tabular}{|c|c|c|c|c|c|c|c|c|c|}
\hline \multirow[b]{2}{*}{$N$} & \multicolumn{3}{|c|}{ Peak height $\left(\mathrm{eV}^{-1} \AA^{-2}\right)$} & \multicolumn{3}{|c|}{ Width $\Gamma(\mathrm{eV})$} & \multicolumn{3}{|c|}{ Peak/total strength (\%) } \\
\hline & $(Q, Q)$ & $(0, H)_{1}$ & $(0, H)_{2}$ & $(Q, Q)$ & $(0, H)_{1}$ & $(0, H)_{2}$ & $(Q, Q)$ & $(0, H)_{1}$ & $(0, H)_{2}$ \\
\hline 1 & 27.5 & 38.2 & 45.6 & $1.73 \times 10^{-2}$ & $1.49 \times 10^{-2}$ & $1.33 \times 10^{-2}$ & 85.8 & 73.7 & 75.5 \\
\hline 2 & 165 & 247 & 328 & $2.42 \times 10^{-3}$ & $2.07 \times 10^{-3}$ & $1.68 \times 10^{-3}$ & 71.6 & 66.1 & 68.4 \\
\hline 3 & 1026 & 1609 & 2383 & $3.81 \times 10^{-4}$ & $3.16 \times 10^{-4}$ & $2.33 \times 10^{-4}$ & 70.5 & 66.0 & 69.1 \\
\hline
\end{tabular}

genvalue but rather the transition strength to the CBS. We have systematically examined the strength in the peak region where about $70 \%$ is concentrated, and have derived a sum rule, within the conduction-band-only model, that explains the integrated transition strength from the ground state to the continuum. Finally, we have identified the factors that allow one to improve the confinement of a continuum bound state, and proposed a way of testing this.

\section{ACKNOWLEDGMENTS}

We are grateful to NSERC-Canada for research Grant No. SAPIN-3198 (D.W.L.S.) and Summer Research Awards (P.J., J.S.), and to DGES-Spain for continued support through Grant No. PB97-0915 (J.M.). This work was carried out as part of CERION, an Esprit Project No. EP-27119 funded by the EU and NSERC.

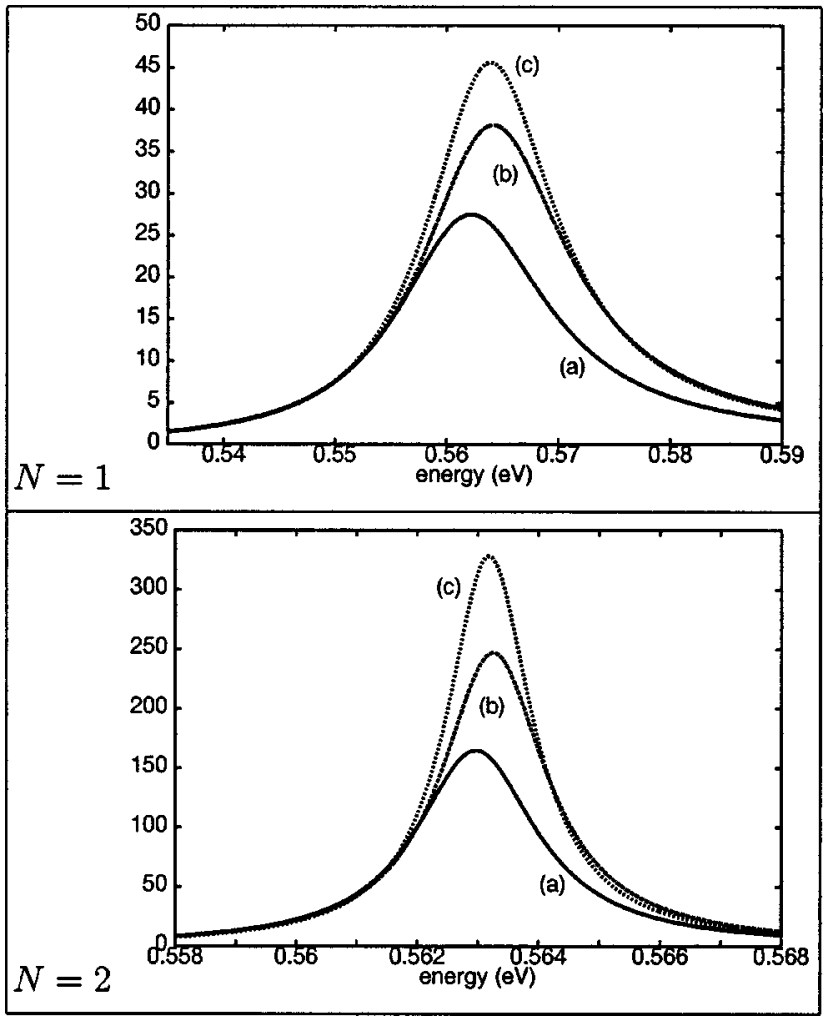

FIG. 9. Transition strength to continuum (units $\mathrm{eV}^{-1} \AA^{-2}$ ) for (a) the case $(Q, Q),\left(\right.$ b) $(0, H)_{1}$, and (c) $(0, H)_{2}$, showing the evolution of the CBS peak. Note change of scale between the upper panel for the $N=1$ reflector and the lower panel for $N=2$.

\section{APPENDIX A: SUM RULE FOR INTRASUBBAND TRANSITIONS}

Sirtori et al. ${ }^{13}$ discussed the sum rule for excitations to the CBS within a two-band Kane model. Here we limit our discussion to what can be done within a conduction-band-only model, Eq. (1). The difficulty which arises in this context is that having an energy-dependent effective mass means that the Hamiltonian does not have a complete orthonormal set of excited states, so the sum rule can only be approximate.

We take the operator to be

$$
-i \hbar S=\left(p \frac{1}{m_{E}}+\frac{1}{m_{0}} p\right)
$$

[cf. Eq. (14). For brevity we drop the * on the effective masses in this Appendix.] Then the sum rule is

$$
\begin{aligned}
\hbar^{2} M_{2}= & \hbar^{2}\left\langle 0\left|S^{\dagger} S\right| 0\right\rangle \\
= & \left\langle 0\left|\left(p \frac{1}{m_{0}}+\frac{1}{m_{E}} p\right)\left(p \frac{1}{m_{E}}+\frac{1}{m_{0}} p\right)\right| 0\right\rangle \\
= & \left\langle 0\left|p \frac{1}{m_{0}} \frac{1}{m_{0}} p\right| 0\right\rangle+\left\langle 0\left|p \frac{1}{m_{0}} \frac{1}{m_{E}} p\right| 0\right\rangle \\
& -i \hbar\left\langle 0\left|p \frac{1}{m_{0}}\left(\frac{1}{m_{E}}\right)^{\prime}\right| 0\right\rangle+\left\langle 0\left|p \frac{1}{m_{E}} \frac{1}{m_{0}} p\right| 0\right\rangle \\
& +i \hbar\left\langle 0\left|\left(\frac{1}{m_{E}}\right)^{\prime} \frac{1}{m_{0}} p\right| 0\right\rangle+\left\langle 0\left|p \frac{1}{m_{E}} \frac{1}{m_{E}} p\right| 0\right\rangle \\
& +i \hbar\left\langle 0\left|\left(\frac{1}{m_{E}}\right)^{\prime} \frac{1}{m_{E}} p\right| 0\right\rangle-i \hbar\left\langle 0\left|p \frac{1}{m_{E}}\left(\frac{1}{m_{E}}\right)^{\prime}\right| 0\right\rangle \\
& +\hbar^{2}\left\langle 0\left|\left(\frac{1}{m_{E}}\right)^{\prime}\left(\frac{1}{m_{E}}\right)^{\prime}\right| 0\right\rangle, \\
M_{2}= & \left.\left|\psi_{0}^{\prime}\right|\right|^{2}\left(\frac{1}{m_{0}}+\frac{1}{m_{E}}\right)^{2} d x+\left\langle 0\left|\left(\frac{1}{m_{E}}\right)^{\prime}\left(\frac{1}{m_{E}}\right)^{\prime}\right| 0\right\rangle \\
& +2 \int \psi_{0}^{\prime}\left(\frac{1}{m_{0}}+\frac{1}{m_{E}}\right)\left(\frac{1}{m_{E}}\right)^{\prime} d x .
\end{aligned}
$$

To obtain this expression we moved the $p$ operators until they act on the ground-state wave function directly. In the case of a constant effective mass, $m_{0}=m_{E}=1$, only the first integral survives. In this case the sum rule must be exact, and we found close agreement between the sum-rule expression, and direct integration: 


$$
M_{2}=\int\left\langle\psi_{0}\left|S^{\dagger}\right| \psi_{E}\right\rangle \frac{d k}{d E}\left\langle\psi_{E}|S| \psi_{0}\right\rangle d E,
$$

where

$$
\frac{d k}{d E}=\frac{1}{2 k} \frac{2 m}{\hbar^{2}} m_{0 b}\left[1+\frac{2\left(E-V_{b}\right)}{E_{b}}\right],
$$

which includes nonparabolicity. The wave number $k$ is defined by the energy above the barrier. At high energy the density of states tends to a constant, rather than going to zero, as it would for constant mass. The case without energy dependence can be recovered if $E_{b} \rightarrow \infty$.

When we introduce $x$ dependence to the effective mass, the terms involving the derivative of $\left(1 / m_{E}^{*}\right)$ contribute. When the mass is piecewise constant, the derivative is a Dirac delta function times the discontinuity in $\left(1 / m_{E}^{*}\right)$. The integral in the last line of Eq. (A3) is then a sum of values evaluated at the layer edges.

When we introduce energy dependence as well, both here, and in the first integral, factors such as $\psi_{0}^{\prime} / m_{E}$ are discontinuous, because the mass $m_{E}$ is taken at one energy and the ground-state wave function at another. To resolve this ambiguity we took the average of the two values on either side of the discontinuity. For these materials, the well and barrier masses are similar, so it is not a large uncertainty. This is the stage at which the sum rule can only be approximate. Moreover, we need a prescription for the energy $E$ at which we evaluate $m_{E}$. Thinking in terms of the doorway state approximation, initially we took the CBS energy. Then we defined an effective doorway energy.

The second term in Eq. (A3) involves the square of a Dirac delta function, and is undefined. We simply omit this contribution.

\section{APPENDIX B: EQUATION (25)}

The stated conditions lead to

$$
\begin{aligned}
& W_{12}^{d}=W_{11}^{b} W_{12}^{a}+W_{12}^{b} W_{22}^{a}=0 \\
& \text { or } \frac{W_{11}^{b}}{W_{12}^{b}}=-\frac{W_{22}^{a}}{W_{12}^{a}} \\
& W_{22}^{d}=W_{21}^{b} W_{12}^{a}+W_{22}^{b} W_{22}^{a}=\lambda=\left(W_{21}^{b}+W_{22}^{b} \frac{W_{22}^{a}}{W_{12}^{a}}\right) W_{12}^{a} \\
& =\left(W_{21}^{b}-W_{22}^{b} \frac{W_{11}^{b}}{W_{12}^{b}}\right) W_{12}^{a}=\left(W_{21}^{b} W_{12}^{b}-W_{22}^{b} W_{11}^{b}\right) \frac{W_{12}^{a}}{W_{12}^{b}}, \\
& \lambda=-\frac{W_{12}^{a}}{W_{12}^{b}}=-\frac{w_{a}(a)}{w_{b}(b)} .
\end{aligned}
$$

In the last step we have used the form of $W$ as in Eq. (2).
${ }^{1}$ F. Capasso, C. Sirtori, J. Faist, D.L. Sivco, S-N.G. Chu, and A.Y. Cho, Nature (London) 358, 565 (1992); C. Sirtori et al., Appl. Phys. Lett. 61, 898 (1992).

${ }^{2}$ G. Lenz and J. Salzman, Appl. Phys. Lett. 56, 871 (1990).

${ }^{3}$ T.A. Weber, Solid State Commun. 90, 713 (1994).

${ }^{4}$ B. Sung, H.C. Chui, E.L. Martinet, and J.S. Harris, Jr., Appl. Phys. Lett. 68, 2720 (1996).

${ }^{5}$ D. Indjin, V. Milanović, and Z. Ikonić, Phys. Rev. B 52, 16762 (1995); Superlattices Microstruct. 23, 225 (1998).

${ }^{6}$ Xue-Hua Wang, Ben-Yuan Gu, Guo-Zhen Yang, and Jian Wang, Phys. Rev. B 58, 4629 (1998).

${ }^{7}$ Wm. Shockley, Phys. Rev. 59, 319 (1939).

${ }^{8}$ D.W.L. Sprung, Hua Wu, and J. Martorell, Am. J. Phys. 61, 1118 (1993).

${ }^{9}$ D.W.L. Sprung, J.D. Sigetich, Hua Wu, and J. Martorell, Am. J. Phys. 68, 715 (2000).
${ }^{10}$ D.F. Nelson, R.C. Miller, and D.A. Kleinman, Phys. Rev. B 35, 7770 (1987)

${ }^{11}$ R.P. Leavitt, Phys. Rev. B 44, 11270 (1991).

${ }^{12}$ T.M. Kalotas and A.R. Lee, Eur. J. Phys. 16, 119 (1995).

${ }^{13}$ C. Sirtori, F. Capasso, J. Faist, and S. Scandolo, Phys. Rev. B 50, 8663 (1994).

${ }^{14}$ R. Kucharczyk*r, M. Steślicka, A. Akjouj, B. Djafari-Rouhani, L. Dobrzynski, and E.H. Boudouti, Phys. Rev. B 58, 4589 (1998).

${ }^{15}$ V. Swaminathan and A.T. Macrander, Materials Aspects of GaAs and InP Based Structures (Prentice-Hall, Englewood Cliffs, NJ, 1991), pp. 1-42.

${ }^{16} \mathrm{~S}$. Adachi, Physical Properties of III-V Semiconductor Compounds (Wiley Interscience, New York, 1992), pp. 96-99.

${ }^{17}$ John H. Davies, Physics of Low-dimensional Semiconductors (Cambridge University, Cambridge, England, 1998). 